\title{
Safety and Efficacy of Oral Physostigmine in the Treatment of Alzheimer Disease
}

\author{
$* \ddagger \S$ Mary Sano, * $\ddagger$ Karen Bell, * $\ddagger \S$ Karen Marder, *Laurie Stricks, \\ $* \dagger \neq \S$ Yaakov Stern, and $* \uparrow \ddagger \S$ Richard Mayeux \\ Departments of ${ }^{*}$ Neurology and $+P$ sychiatry, Columbia University, College of \\ Physicians and Surgeons, $¥$ The New York State Psychiatric Institute Memory Disorders \\ Clinic, and \$Center for Alzheimer's Disease Research, New York, New York, U.S.A.
}

\begin{abstract}
Summary: Results of therapeutic trials with physostigmine in the treatment of Alzheimer disease (AD) have been inconsistent and controversy persists concerning safety and efficacy. In a double-blind, placebo-controlled, crossover study, patients received 6 weeks of oral physostigmine (OP) and placebo in random order. Twenty-nine patients with $\mathrm{AD}$ received as much as $16 \mathrm{mg} / \mathrm{day}$ of OP and were assessed with neuropsychological and functional measures. No significant cardiac side effects were noted, though other systemic adverse effects were noted, requiring dose reduction in four patients. There was a slight but significant improvement $(12 \%)$ in performance on the selective reminding test with physostigmine and the memory performance was correlated with dosage. This improvement compares favorably with the $15 \%$ decrease in scores seen in an untreated comparison cohort followed for an equivalent time period. There was a trend toward an improvement in communication and a reduction in memory complaint. These results suggest that oral physostigmine is safe and may improve memory in AD. Key Words: Alzheimer diseasePhysostigmine-Safety-Efficacy.
\end{abstract}

Oral physostigmine (OP), a cholinesterase inhibitor, has frequently been used as an experimental treatment for memory and cognitive loss in probable Alzheimer disease (AD). The results of these studies have been inconsistent, due in part to methodological differences. Initially, investigators used short dose-finding periods to determine the most effective dose based on memory performance $(1,2)$. This dose-finding procedure may have maximized side effects at low doses while longer treatment may allow tolerance to develop (3). Furthermore, short-term exposure was not useful in predicting the improvement found in a longer study (4-6).

Address correspondence and reprint requests to Dr. M. Sano at Neurological Institute, 710 West 168th Street, New York, NY 10032, U.S.A. 
To determine the efficacy of physostigmine in patients without prior exposure to the drug, we conducted a trial in patients with $\mathrm{AD}$ using a double-blind, placebo-controlled, crossover design. A dose-titration period was used to maximize exposure to drug and testing procedures but was not used to determine an effective dose. Cognitive and functional assessments at a maximum dose of $16 \mathrm{mg} /$ day were made after a period of 6 weeks. Cardiac and systemic side effects were monitored to assess safety.

\section{METHODS}

Subjects

Twenty-nine patients who met NINCDS-ADRDA research diagnostic criteria for probable or possible $\mathrm{AD}$ (7) gave informed consent to participate in the study. Subjects were living at home with varying degrees of supervision throughout the study period. Each had an identified caregiver (usually a spouse or child) who acted as an advocate for the patient in the matter of informed consent.

\section{Procedures}

The patients were initially admitted to the General Clinical Research Center (GCRC) at Columbia Presbyterian Medical Center. On the day of admission, the Selective Reminding Test (SRT) was administered as a baseline measure.

\section{Phase One: Titration}

This phase lasted 2 days. Patients received five different doses of OP, ranging from 2 to $4 \mathrm{mg}$ in $0.5 \mathrm{mg}$ increments. Each day began with a dose of $2 \mathrm{mg}$. This was followed by four other doses at $2 \mathrm{~h}$ intervals, administered in random order. The SRT was administered six times during this period using a different equivalent form each time. This procedure was designed to familiarize the subject with test taking. The highest dose tolerated without serious side effect was chosen for each patient.

\section{Phase Two: Double Blind}

Double-blind random assignment was used to determine the order of drug and placebo assignment (i.e., drug followed by placebo or placebo followed by drug). Drug and placebo were administered for 6 weeks. Drug A (OP or placebo) was administered at $2 \mathrm{~h}$ intervals, four times a day at the dose chosen in phase 1 . Patients were discharged if they were free of side effects after receiving the initial two doses. They continued to take the medication under supervision at home for 6 weeks. At the end of the 6 week interval, patients returned to the GCRC and underwent memory testing $30 \mathrm{~min}$ after drug administration. This was done twice (after the 0900 and $1300 \mathrm{~h}$ doses) and the average of the two scores was recorded. Routine serum chemistries were drawn. An electrocardiogram (ECG) and other outcome measures (described below) were also completed at this time. 


\section{Phase Three: Double-Blind Crossover}

On the day following the end of phase two, the patients received the first two doses of the alternate medication and were discharged if they were symptom free. They continued medication according to the same schedule used in phase two. Patients returned to the GCRC and assessment procedures identical to those in phase two were repeated.

\section{Medication}

Physostigmine (physostigmine salicylate), supplied in $1 \mathrm{mg}$ tablets, and identical placebo was provided by the Forest Pharmaceuticals Inc., a subsidiary of Forest Laboratories Inc. (St. Louis, MO, U.S.A.).

\section{Outcome Measures}

\section{Primary Outcome Measure}

For comparability with other studies $(1,2,4,5)$, the primary outcome measure was performance on the SRT. In this 12 item, 12 trial, verbal memory test, modified from Buschke and Fuld (8), three measures are derived: Total Recall (the total number of words recalled), Long Term Retrieval (the number of words recalled without reminding), and Intrusions, words recalled that are not on the list (each occurrence after a single correction). Incidental recall is assessed $15 \mathrm{~min}$ later. A recognition trial follows, in which the subject discriminates words on the list from distractor words. Different, equivalent forms of this test were used at each administration. Additional details of the administration and scoring procedure were previously described $(1,4)$.

\section{Other Outcome Measures}

Independent function was assessed with two instruments that were completed by the caregiver: The Sickness Impact Profile (SIP) (9), a standardized questionnaire that measures the impact of illness on functional abilities in the areas of Sleep and Rest, Home Management, Recreation and Pastimes, Physical Activities (including ambulation, mobility, body care, and movement), and Psychosocial Activities (including social interaction, alertness behavior, emotional behavior, and communication), yields a total score and subscale scores for each area. The Squire's Memory Questionnaire (SMQ) (10) measures memory in daily activities.

\section{Cardiac and Side Effect Evaluation}

Patients received baseline ECGs prior to receiving physostigmine in the titration phase. At the end of each 6 week interval, an ECG was performed $30 \mathrm{~min}$ after administration of medication. Systemic side effects (such as nausea, vomiting, and diaphoresis) were also recorded for each phase based on a review of nursing notes and interviews with the patient and/or caregiver. 


\section{Data Analysis}

The effect of OP on the SRT was assessed using paired $t$ tests. A two-way analysis of variance was used to examine treatment and order effects. Drug and placebo performance differences were also examined for the neuropsychological and independent function measures using paired $t$ tests. In addition, correlations between dose, demographics, and outcome measures were examined.

\section{RESULTS}

\section{Description of Subjects}

The mean age \pm SD of the patients was $69.1 \pm 9.1$ years with an average duration of illness of $4.2 \pm 0.3$ years. The mean score on the Modified MiniMental State Examination (mMMSE) was $35.65 \pm 7.22$. This is equivalent to an 18 on the of Mini-Mental State Examination (MMSE) described by Folstein et al. $(11,12)$.

\section{Primary Outcome Measures}

The mean values for the SRT scores for the drug and placebo conditions are given in Table 1. There was a significant difference between the drug and placebo condition in the Total Recall $(p<0.01)$ and the Intrusion scores $(p<0.01)$. There were no significant differences in the other SRT scores.

Table 2 presents the results of a two-way analysis of variance for the Total Recall and Intrusion scores to examine the effect of treatment (drug vs. placebo) and order (drug first vs. placebo first). There was a significant main effect for drug $(F=5.30, p<0.05)$. The effect of order and the interaction of drug and order were not significant.

The average total daily dosage was $13.11 \pm 2.31 \mathrm{mg}$. There was a small but significant correlation between dose and change in the SRT Total score $(r=0.48$; $p<0.05$ ). Change in SRT Total score did not correlate with mMMSE score at baseline, age, or duration of illness.

\section{Other Outcome Measures}

There was no significant change between the drug and placebo conditions for the SIP total score. The communications subscale demonstrated a significant

TABLE 1. Performance on the Selective Reminding Test (SRT) (mean $\pm S D)$ in patients with Alzheimer disease

\begin{tabular}{lccc}
\hline \multicolumn{1}{c}{ SRT measures } & Physostigmine & Placebo & $\begin{array}{c}\text { Physostigmine/placebo } \\
\text { difference }\end{array}$ \\
\hline Total Recall & $57.1 \pm 20.9$ & $52.9 \pm 21.1$ & $4.2 \pm 7.7^{*}$ \\
Long Term Recall & $29.6 \pm 18.9$ & $26.8 \pm 17.9$ & $2.8 \pm 9.8$ \\
Intrusions & $5.4 \pm 5.8$ & $8.5 \pm 8.6$ & $-3.0 \pm 6.5^{*}$ \\
Delayed Recall & $1.0 \pm 1.7$ & $1.2 \pm 1.9$ & $-0.2 \pm 2.2$ \\
Delayed Recognition & $7.7 \pm 2.8$ & $7.5 \pm 2.8$ & $0.2 \pm 1.7$ \\
\hline
\end{tabular}

* Significant difference between drug and placebo, $p<0.01$. 
TABLE 2. Two-way analysis of variance of the total recall and intrusion score from the selective reminding test to examine the effect of order and treatment (physostigmine vs. placebo)

\begin{tabular}{lrrrr}
\hline & df & \multicolumn{1}{c}{ SS } & $F$ ratio & $p$ value \\
\hline Total recall score & & & & \\
source of variation & 28 & $23,871.4$ & 28.44 & \\
$\quad$ Patients & 1 & 26.0 & 0.87 & 0.36 \\
$\quad$ Order & 1 & 271.1 & 9.04 & \\
$\quad$ Treatment & 27 & 809.5 & & \\
$\quad$ Residual & 57 & $24,978.0$ & & \\
$\quad$ Total & & & 4.30 & 0.16 \\
Intrusion score & 28 & $2,426.9$ & 2.05 & 0.03 \\
source of variation & 1 & 41.3 & 5.30 & \\
$\quad$ Patients & 1 & 106.9 & & \\
$\quad$ Order & 27 & 544.2 & & \\
$\quad$ Treatment & 57 & 3119.3 & & \\
$\quad$ Residual & & &
\end{tabular}

SS, sum of squares.

improvement $(p<0.01)$ for the drug condition. No other subscale changed significantly.

The improvement for drug condition in the overall SMQ score reached borderline significance $(p<0.06)$; there were no differences in the individual items on this scale.

\section{Cardiac and Systemic Side Effects}

Eight patients had ECG abnormalities at baseline: one had left bundle branch block, one had left anterior fascicular block, one had left inferior wall infarct, one had atrial fibrillation, one had first-degree atrioventricular block, one had left atrial enlargement, one had premature supraventricular complexes, and one had nonspecific T-wave abnormalities.

Eight patients had sinus bradycardia (three had borderline values of 59 beats/ $\min$ ). Five patients had sinus bradycardia in both drug and placebo conditions. Three patients had sinus bradycardia in the drug condition only and one in the placebo condition only. There was no association between dosage and heart rate or the presence of any other cardiac abnormality.

The frequency of systemic side effects is described in Table 3. Fifteen (52\%) patients experienced systemic side effects during the titration phase of the study.

TABLE 3. Frequency of reported systemic side effects in each phase of the study

\begin{tabular}{lccc}
\hline Symptom & Titration phase & Active drug & Placebo \\
\hline Nausea & 12 & 2 & 2 \\
Vomiting & 6 & 0 & 1 \\
Diaphoresis & 2 & 0 & 0 \\
Diarrhea & 1 & 1 & 1 \\
Dizziness & 0 & 1 & 1 \\
Headache & 0 & 0 & 1 \\
Total number with any side effect & 15 & 4 & 4 \\
\hline
\end{tabular}


Twelve of the patients experienced nausea and 6 vomited. Other systemic side effects were dizziness, diaphoresis, and diarrhea. Four patients had their medication dosage decreased in the initial few weeks of the double-blind phase because of side effects.

Despite dosage adjustments in the titration period, five patients experienced intermittent adverse symptoms in the double-blind phases. Two patients had nausea and diarrhea and one had dizziness on both drug and placebo. One patient had intermittent headaches only on OP and one had nausea and vomiting only on placebo.

There were no clinically significant changes in blood counts, liver enzymes, or other chemistry results. Chloride levels were increased in six patients: two on drug only and four on drug and placebo. Alkaline phosphatase levels were elevated in two patients: one on drug only and one on both drug and placebo. These elevations were not considered clinically significant.

\section{Post Hoc Analyses to Evaluate the Effect on SRT}

To evaluate the effect seen on the SRT, retrospective comparisons were made with a cohort of patients with $\mathrm{AD}$, described elsewhere (13), receiving placebo for a period of 6 months. For this comparison, the interim SRT scores ( 3 months, i.e., 12 weeks) were examined since that was the time interval of the entire crossover period. These patients received a six-trial version of the SRT; thus, all scores were converted to percent of baseline to make comparisons. In the current study, 28 of the 29 patients had baseline SRT scores. The SRT Total Score increased $12 \%$ over baseline during the OP phase compared to a $1 \%$ increase on placebo. The size of the change varied with the order in which drug and placebo were administered but significantly better performance was observed with drug than with placebo. In the previously described cohort (13), the SRT Total Score demonstrated a $15 \%$ decrease from baseline during a 3 month interval. The change scores for both groups are illustrated in Fig. 1 .

\section{DISCUSSION}

The use of physostigmine for improving memory and cognition in patients with AD has been frequently studied and there is a small but significant improvement in formal memory testing $(1-5,14)$. These studies lend support to cholinesterase inhibition as a mechanism for treatment of memory loss in AD. The present study also demonstrates statistically significant improvement in memory testing with OP in patients with $\mathrm{AD}$. Overall, the group demonstrated a $12 \%$ improvement over baseline in memory scores. While the exact clinical meaningfulness of this change is difficult to assess, it is known that memory performance deteriorates with disease progression in AD. For example, a previous study found that SRT Total Recall score decreased $15 \%$ in 3 months and $21 \%$ in 6 months (13). The retrospective nature of the comparison limits the conclusions that can be drawn. However, the improvement seen with OP may be meaningful.

There was little change in the functional scales used in this study. The improve- 
\% Change in SRT Total Score

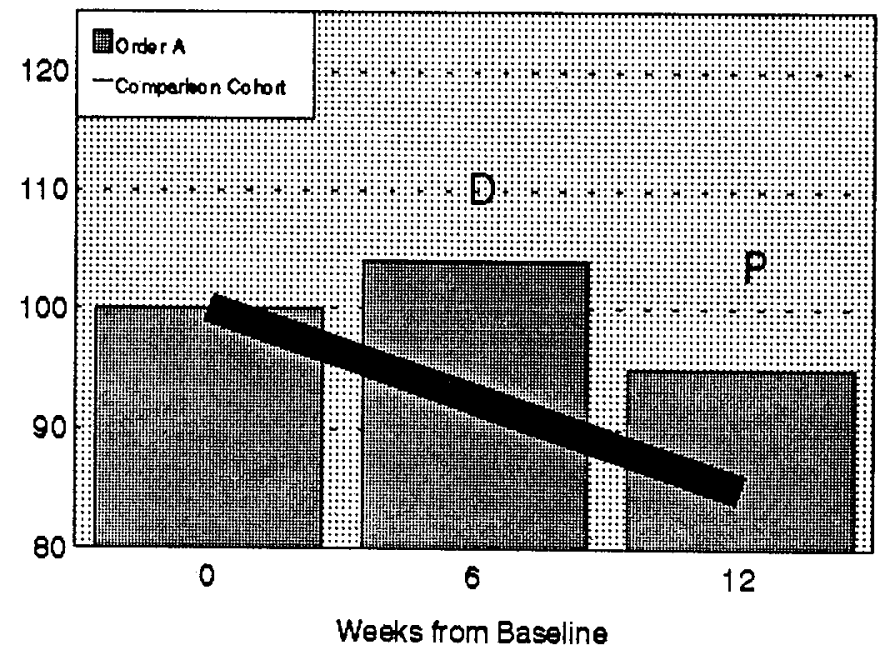

\% Change in SRT Total Score
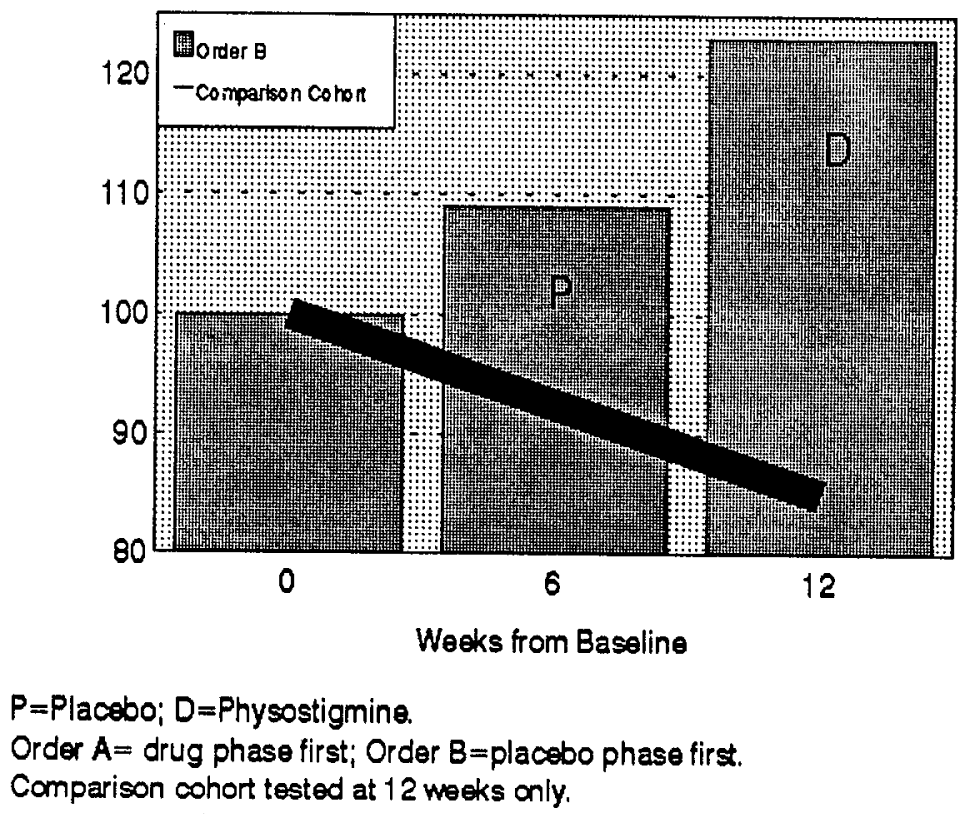

FIG. 1. Selective reminding test: total score. Change from baseline in SRT total score in patients with Alzheimer disease with physostigmine treatment and in a comparison cohort. Bar graph describes effect of physostigmine; line graph describes a placebo-treated comparison cohort.

ment in the communication subscales of the SIP and a trend toward an improvement in the SMQ, a subjective measure of memory complaint, must be viewed with caution given the multiple comparisons that were conducted on these measures. These findings suggest other domains that might be assessed in clinical 
trials to determine if statistical improvement in memory test scores reflect a clinically meaningful effect.

There was a significant relationship between improvement on memory testing and dose of OP. This relationship has not been demonstrated before, although previous studies have used lower doses, perhaps because of concerns about cardiac side effects. Intravenous administration of physostigmine for confusion, disorientation, and coma following anesthesia has been associated with sinus bradycardia (due to increased vagal tone at the sinoatrial node), atrial fibrillation (15), ventricular tachycardia, and premature ventricular contractions (16). In the present study, ECG findings suggests that at these doses there was no significant change in cardiac status, even in patients with baseline abnormalities. Systemic side effects such as nausea and vomiting are well known with OP (3) and though these are thought to be a less serious threat, they represented the limiting factor in this study. The dose was reduced at the first report of side effect. No attempt was made to reinitiate a higher dose or to treat the side effect. This strategy limited the size of doses, which might have also limited efficacy. Given the relative safety demonstrated in this study, further investigation at higher doses should be considered.

The limited improvements may also be due to the specific agent, physostigmine. Arnal et al. have described a centrally active cholinesterase inhibitor (an analogue of pyridostigmine, norpyridostigmine) that is effective at doses that have less side effects than physostigmine in animal models (17). Behavioral studies in animals have demonstrated that norpyridostigmine improves accuracy in a maze learning task in a dose-related fashion (18). In addition, other cholinesterase inhibitors with longer duration of action have been examined in animal models. Perhaps other cholinesterase inhibitors, with central nervous system specificity and minimal peripheral activation, would prove to be useful agents.

Acknowledgment: The authors gratefully acknowledge the contributions of Drs. Lucien Coté and George Dooneief for assistance in preparing this manuscript. We also thank Ms. Edith Flaster and Mr. Donald McMahon for their assistance with the statistical analysis. This work was supported by federal grants AG-08702 and RR00645 to the Clinical Research Center in the Presbyterian Hospital in the City of New York and The Charles S. Robertson Memorial gift for Research in Alzheimer's Disease. Dr. Sano is a Herbert Irving Assistant Professor in the Department of Neurology.

\section{REFERENCES}

1. Stern Y, Sano M, Mayeux R. Effects of oral physostigmine in Alzheimer's disease. Ann Neurol 1987;22:306-10.

2. Thal LJ, Fuld PA, Masur DM, Sharpless NS. Oral physostigmine and lecithin improve memory in Alzheimer's Disease. Ann Neurol 1983;13:491-6.

3. Taylor P. Anticholinesterase agents. In: Gilman A, Goodman L, Gilman A, eds. Goodman and Gilmans the pharmacological basis of therapeutics, sixth edition. New York: Macmillan Publishing Co. Inc., 1985:100-19.

4. Stern Y, Sano M, Mayeux R. Long-term administration of oral physostigmine in Alzheimer's disease. Neurology 1988;38:1837-41.

5. Beller SA, Overall JE, Swann AC. Efficacy of oral physostigmine in primary degenerative dementia. Psychopharmacology 1985;87:147-51. 
6. Beller SA, Overall JE, Rhoades HM, Swann AC. Long-term out-patient of treatment of senile dementia with oral physostigmine. J Clin Psychiatry 1988;49:400-4.

7. McKhann G, Drachman D, Folstein M, Katzman R, Price D, Stadlan EM, Clinical Diagnosis of Alzheimer's Disease NINCDS-ADRDA Work Group. Neurology 1984;34:939-44.

8. Buschke H, Fuld PA. Evaluating storage, retention and retrieval in disordered memory and learning. Neurology 1974;24:1019-25.

9. Bergner M, Bobbitt R, Carter W, Gilson B. The sickness impact profile: development and final revision of a health status measure. Med Care 1981;19:787-805.

10. Squire LR, Wetzel CD, Slater PC. Memory complaint after ECT, assessment with a new selfrating instrument. Biol Psychiatry 1979;14:791-801.

11. Folstein MF, Folstein SE, McHugh PR. "Mini-Mental State." A practical method for grading the cognitive state of patients for the clinician. J Psychiatr Res 1975;12:189-98.

12. Stern Y, Sano M, Paulson J, Mayeux R. Modified Mini-Mental State Examination: validity and reliability. Neurology 1987;37(suppl 1):179.

13. Sano M, Bell K, Cote L, et al. A double-blind parallel design pilot study with acetyl-1-carnitine in patients with probable Alzheimer's disease. Arch Neurol 1992;49:1137-41.

14. Mohs RC, Davis BM, Johns CA, et al. Oral physostigmine treatment of patients with Alzheimer's disease. Am J Psychiatry 1985;142:28-33.

15. Bidwai AV, Cornelius LR, Stanley TH. Reversal of innovar-induced postanesthetic somnolence and disorientation with physostigmine. Anesthesiology 1976;441:249-52.

16. Levy R. Arrythmias following physostigmine administration in Jimson weed poisoning. JACEP 1977;6:107.

17. Arnal F, Coté LJ, Ginsburg S, Lawrence GD, Naini A, Sano M. Studies on centrally active and reversible acetylcholinesterase inhibitors. Neurochem Res 1990;15:593-7.

18. Bach ME, Sano M, Coté LJ, Ginsburg S, Mayeux R. Learning deficits in rats are attenuated by a novel acetylcholinesterase inhibitor 3-pyridinol dimethylcarbamate. Soc Neurosci Abstr 1990; $16: 58.16$. 